\title{
AN ANALOGUE OF SOME INEQUALTTIES OF P. TURÁN CONCERNING ALGEBRAIC POLYNOMIALS HAVING ALL ZEROS INSIDE $[-1,+1]$. II
}

\author{
A. K. VARMA \\ (In memory of Professor P. Turán)
}

ABSTRACT. Let $P_{n}(x)$ be an algebraic polynomial of degree $<n$ having all zeros inside $[-1,+1]$; then we have

$$
\int_{-1}^{1} P_{n}^{\prime 2}(x) d x>\left(\frac{n}{2}+\frac{3}{4}+\frac{3}{4 n}\right) \int_{-1}^{1} P_{n}^{2}(x) d x .
$$

This bound is much sharper than found in [2]. Moreover, if $P_{n}(1)=P_{n}(-1)$

$=0$, then under the above conditions we have

$$
\int_{-1}^{1} P_{n}^{\prime 2}(x) d x>\left(\frac{n}{2}+\frac{3}{4}+\frac{3}{4(n-1)}\right) \int_{-1}^{1} P_{n}^{2}(x) d x,
$$

equality for $P_{n}(x)=\left(1-x^{2}\right)^{m}, n=2 m$.

1. Let $H_{n}$ be the set of all polynomials whose degree is $n$ and whose zeros are all real and lie inside $[-1,+1]$. Throughout this paper $x_{1}, x_{2}, \ldots, x_{n}$ will denote the roots of $P_{n}(x)\left(P_{n} \in H_{n}\right)$. In an earlier work [2] the following theorem was proved.

TheOREM A. Let $P_{n} \in H_{n}$; then we have

$$
\begin{aligned}
2 \int_{-1}^{1} P_{n}^{\prime 2}(x) d x & >2 \int_{-1}^{1}\left(1-x^{2}\right) P_{n}^{\prime 2}(x) d x \\
& =n \int_{-1}^{1} P_{n}^{2}(x) d x+\int_{-1}^{1} P_{n}^{2}(x) \sum_{k=1}^{n} \frac{1-x_{k}^{2}}{\left(x-x_{k}\right)^{2}} d x .
\end{aligned}
$$

Hence, for $P_{n} \in H_{n}$ we have

$$
\left\|P_{n}^{\prime}\right\|_{L_{2}[-1,+1]}>\frac{n^{1 / 2}}{2^{1 / 2}} \cdot\left\|P_{n}\right\|_{L_{2}[-1,+1]} .
$$

$\left(P_{n}^{\prime}(x)\right.$ stands for the derivative of $P_{n}(x)$ ).

(1.2) is analogous to P. Turán's [1] beautiful theorem in the $L_{\infty}$ norm.

In this paper we give a more precise form of (1.2). We prove

THEOREM 1. Let $P_{n} \in H_{n}$ and

$$
P_{n}(1)=P_{n}(-1)=0 \text {; }
$$

Received by the editors February 2, 1977.

AMS (MOS) subject classifications (1970). Primary 26A60, 30A40.

c) American Mathematical Society 1978 
then we have

$$
\left\|P_{n}^{\prime}\right\|_{L_{2}[-1,+1]}^{2}>\left(\frac{n}{2}+\frac{3}{4}+\frac{3}{4(n-1)}\right)\left\|P_{n}\right\|_{L_{2}[-1,+1]}^{2}
$$

with equality for $P_{n}(x)=\left(1-x^{2}\right)^{m}, n=2 m$.

TheOREM 2. Let $P_{n} \in H_{n}$; then we have

(1.5) $2 \int_{-1}^{1} P_{n}^{\prime 2}(x) d x>2 \int_{-1}^{1}\left(1-x^{4}\right) P_{n}^{\prime 2}(x) d x \geqslant \alpha_{1, n} \int_{-1}^{1} P_{n}^{2}(x) d x$,

(1.6) $2 \int_{-1}^{1} P_{n}^{\prime 2}(x) d x>2 \int_{-1}^{1}\left(1-x^{6}\right) P_{n}^{\prime 2}(x) d x \geqslant \alpha_{2, n} \int_{-1}^{1} P_{n}^{2}(x) d x$,

(1.7) $2 \int_{-1}^{1} P_{n}^{\prime 2}(x) d x>2 \int_{-1}^{1}\left(1-x^{8}\right) P_{n}^{\prime 2}(x) d x \geqslant \alpha_{3, n} \int_{-1}^{1} P_{n}^{2}(x) d x$,

where

$$
\begin{aligned}
& \alpha_{1, n}=n+\frac{3 n}{2 n+3}, \quad \alpha_{2, n}=\alpha_{1, n}+\frac{15 n}{(2 n+5)(2 n+3)}, \\
& \alpha_{3, n}=\alpha_{2, n}+\frac{105 n}{(2 n+7)(2 n+5)(2 n+3)} .
\end{aligned}
$$

Equality holds on the right-hand side of $(1.5)-(1.7)$ iff $P_{n}(x)=\left(1-x^{2}\right)^{m}$, $n=2 m$. From (1.7) we obtain $(n \geqslant 3)$

$$
\left\|P_{n}^{\prime}\right\|_{L_{2}[-1,+1]}^{2}>\left(\frac{n}{2}+\frac{3}{4}+\frac{3}{4 n}\right)\left\|P_{n}\right\|_{L_{2}[-1,+1]}^{2}
$$

This result is sharp for $P_{0 n}(x)=\left(1-x^{2}\right)^{m}, n=2 m$; indeed we have

$$
\left\|P_{0 n}^{\prime}\right\|_{L_{2}[-1,+1]}^{2}=\left(\frac{n}{2}+\frac{3}{4}+\frac{3}{4(n-1)}\right)\left\|P_{0 n}\right\|_{L_{2}[-1,+1]}^{2}
$$

2. Some identities. For the proofs of Theorems 1 and 2 the following identities are needed.

IDENTITY 2.1. Let $P_{n}(x)$ be any polynomial of degree $n$; then for all positive integers $r$,

$$
\begin{aligned}
2 \int_{-1}^{1}\left(1-x^{2 r}\right) P_{n}^{\prime 2}(x) d x= & \sum_{i=1}^{r}(n+2 i-1) \int_{-1}^{1} x^{2 i-2} P_{n}^{2}(x) d x \\
& -r(2 r-1) \int_{-1}^{1} x^{2 r-2} P_{n}^{2}(x) d x+\sum_{i=1}^{r} S_{2 i-2}
\end{aligned}
$$

where

$$
S_{2 i}=\int_{-1}^{1} x^{2 i} P_{n}^{2}(x) \sum_{k=1}^{n} \frac{1-x_{k}^{2}}{\left(x-x_{k}\right)^{2}} d x .
$$

Proof. On integrating by parts, we obtain 


$$
\begin{aligned}
\int_{-1}^{1}\left(1-x^{2 r}\right) P_{n}^{\prime}(x) P_{n}^{\prime}(x) d x \\
\quad=-\int_{-1}^{1} P_{n}(x)\left\{\left(1-x^{2 r}\right) P_{n}^{\prime \prime}(x)-2 r x^{2 r-1} P_{n}^{\prime}(x)\right\} d x ;
\end{aligned}
$$

we rewrite it as

$$
\begin{aligned}
2 \int_{-1}^{1}\left(1-x^{2 r}\right) P_{n}^{\prime 2}(x) d x= & \int_{-1}^{1}\left(1-x^{2 r}\right)\left(P_{n}^{\prime 2}(x)-P_{n}(x) P_{n}^{\prime \prime}(x)\right) d x \\
& +2 r \int_{-1}^{1} x^{2 r-1} P_{n}(x) P_{n}^{\prime}(x) d x
\end{aligned}
$$

On using

$$
\begin{gathered}
P_{n}^{\prime 2}(x)-P_{n}(x) P_{n}^{\prime \prime}(x)=P_{n}^{2}(x) \sum_{k=1}^{n} \frac{1}{\left(x-x_{k}\right)^{2}} \\
\frac{\left(1-x^{2}\right)}{\left(x-x_{k}\right)^{2}}=1-\frac{2 x}{x-x_{k}}+\frac{1-x_{k}^{2}}{\left(x-x_{k}\right)^{2}}
\end{gathered}
$$

and

$$
P_{n}^{\prime}(x)=P_{n}(x) \sum_{k=1}^{n} \frac{1}{\left(x-x_{k}\right)}
$$

in (2.3) we obtain

$$
\begin{aligned}
2 \int_{-1}^{1}(1 & \left.-x^{2 r}\right) P_{n}^{\prime 2}(x) d x=2 r \int_{-1}^{1} x^{2 r-1} P_{n}(x) P_{n}^{\prime}(x) d x \\
& +\int_{-1}^{1}\left(\sum_{i=1}^{r} x^{2 i-2}\right)\left\{\sum_{k=1}^{n}\left[1-\frac{2 x}{x-x_{k}}+\frac{\left(1-x_{k}^{2}\right)}{\left(x-x_{k}\right)^{2}}\right] P_{n}^{2}(x)\right\} d x \\
= & n \int_{-1}^{1}\left(\sum_{i=1}^{r} x^{2 i-2}\right) P_{n}^{2}(x) d x+\sum_{i=1}^{r} S_{2 i-2} \\
& +2 \int_{-1}^{1}\left(r x^{2 r-1}-\sum_{i=1}^{r} x^{2 i-1}\right) P_{n}(x) P_{n}^{\prime}(x) d x .
\end{aligned}
$$

On integration by parts we have

$$
\begin{aligned}
& 2 \int_{-1}^{1}\left(r x^{2 r-1}-\sum_{i=1}^{r} x^{2 i-1}\right) P_{n}(x) P_{n}^{\prime}(x) d x \\
& \quad=-\int_{-1}^{1}\left\{(2 r-1) r x^{2 r-2}-\sum_{i=1}^{r}(2 i-1) x^{2 i-2}\right\} P_{n}^{2}(x) d x .
\end{aligned}
$$

Combining (2.7) and (2.8) we obtain (2.1).

IDENTITY 2.2. Let $P_{n}(x)$ be any polynomial of degree $n, r$ any fixed positive integer; then we have 


$$
\begin{aligned}
2 \int_{-1}^{1} x^{2 r-2}(1- & \left.x^{2}\right)^{2} P_{n}^{\prime 2}(x) d x=\left(2 r^{2}+r-n\right) \int_{-1}^{1} x^{2 r} P_{n}^{2}(x) d x \\
& +\left(n+4 r-4 r^{2}-1\right) \int_{-1}^{1} x^{2 r-2} P_{n}^{2}(x) d x+S_{2 r-2}-S_{2 r} \\
& +(r-1)(2 r-3) \int_{-1}^{1} x^{2 r-4} P_{n}^{2}(x) d x
\end{aligned}
$$

where $S_{2 r}$ is defined by (2.2). For $r=1$ the last term on the right-hand side at (2.9) does not appear.

The proof of this identity is similar to (2.1) and so we omit the details.

IDENTITY 2.3. Let $P_{n}(x)$ be any polynomial of degree $n, r$ any fixed positive integer; then we have

$$
\begin{aligned}
(n+r)(2 n+2 r+1) & \int_{-1}^{1} x^{2 r} P_{n}^{2}(x) d x=S_{2 r}-S_{2 r-2} \\
& +2 \int_{-1}^{1} x^{2 r-2}\left(n x P_{n}(x)+\left(1-x^{2}\right) P_{n}^{\prime}(x)\right)^{2} d x \\
& +\left(4 n r+4 r^{2}-4 r-3 n+1\right) \int_{-1}^{1} x^{2 r-2} P_{n}^{2}(x) d x \\
& -(r-1)(2 r-3) \int_{-1}^{1} x^{2 r-4} P_{n}^{2}(x) d x
\end{aligned}
$$

Proof. We write

$$
\begin{aligned}
\int_{-1}^{1} x^{2 r} P_{n}^{2}(x) d x= & \frac{1}{n^{2}} \int_{-1}^{1} x^{2 r-2}\left(n x P_{n}(x)+\left(1-x^{2}\right) P_{n}^{\prime}(x)\right)^{2} d x \\
& -\frac{1}{n^{2}} \int_{-1}^{1} x^{2 r-2}\left(1-x^{2}\right)^{2} P_{n}^{\prime 2}(x) d x \\
& -\frac{2}{n} \int_{-1}^{1} x^{2 r-1}\left(1-x^{2}\right) P_{n}(x) P_{n}^{\prime}(x) d x
\end{aligned}
$$

On using (2.9) and

$$
\begin{aligned}
& \int_{-1}^{1} 2 x^{2 r-1}\left(1-x^{2}\right) P_{n}(x) P_{n}^{\prime}(x) d x \\
& =-\int_{-1}^{1}\left((2 r-1) x^{2 r-2}-(2 r+1) x^{2 r}\right) P_{n}^{2}(x) d x
\end{aligned}
$$

we obtain (2.10) on rearranging (2.11).

From (2.10) and

$$
\int_{-1}^{1} x^{2 r}\left(n x P_{n}(x)+\left(1-x^{2}\right) P_{n}^{\prime}(x)\right)^{2} d x \geqslant 0
$$

(equality for only $P_{n}(x)=\left(1-x^{2}\right)^{m}, n=2 m$, the following inequalities are obtained.

$$
\int_{-1}^{1} x^{2} P_{n}^{2}(x) d x \geqslant \frac{1}{2 n+3} \int_{-1}^{1} P_{n}^{2}(x) d x+\frac{S_{2}-S_{0}}{(n+1)(2 n+3)}
$$


where

where

$$
\begin{aligned}
& \alpha_{4, n}= \frac{5 n+9}{(2 n+5)(2 n+3)(n+2)(n+1)}, \\
& \alpha_{5, n}= \frac{2 n^{2}-6}{(2 n+5)(2 n+3)(n+2)(n+1)}, \\
& \alpha_{6, n}=\frac{1}{(2 n+5)(n+2)} \\
& \int_{-1}^{1} x^{6} P_{n}^{2}(x) d x \geqslant \frac{15}{(2 n+7)(2 n+5)(2 n+3)} \int_{-1}^{1} P_{n}^{2}(x) d x \\
& \\
&-\alpha_{7, n} S_{0}-\alpha_{8, n} S_{2}-\alpha_{9, n} S_{4},
\end{aligned}
$$$$
\alpha_{7, n}=\frac{33 n^{2}+152 n+165}{(2 n+7)(2 n+5)(2 n+3)(n+3)(n+2)(n+1)},
$$$$
\alpha_{8, n}=\frac{18 n^{3}+62 n^{2}-90}{(2 n+7)(2 n+5)(2 n+3)(n+3)(n+2)(n+1)},
$$$$
\alpha_{9, n}=\frac{2 n^{2}-15}{(2 n+7)(2 n+5)(n+3)(n+2)} \text {. }
$$

IDENTITY 2.4. Let $P_{n}(x)$ be any polynomial of degree $n$; then we have

$$
\begin{aligned}
& 2(n+3) \int_{-1}^{1} x^{2} P_{n}^{2}(x) d x-(2 n+5) \int_{-1}^{1} x^{4} P_{n}^{2}(x) d x-\int_{-1}^{1} P_{n}^{2}(x) d x \\
&= \frac{2}{(n+2)} \int_{-1}^{1}\left(1-x^{2}\right)\left(n x P_{n}(x)+\left(1-x^{2}\right) P_{n}^{\prime}(x)\right)^{2} d x \\
&-\frac{1}{(n+2)} \int_{-1}^{1}\left(1-x^{2}\right)^{2} \sum_{k=1}^{n} \frac{\left(1-x_{k}^{2}\right)}{\left(x-x_{k}\right)^{2}} P_{n}^{2}(x) d x .
\end{aligned}
$$

Proof. On using (2.3) for $r=2$, and $r=1$ on the left-hand side of (2.19) gives the result.

Replacing $n$ in the above identity by $n-2$, and $P_{n}(x)$ by $q_{n-2}(x)$, we obtain $(n \geq 2)$

$$
\begin{array}{r}
2 n \int_{-1}^{1} x^{2}\left(1-x^{2}\right) q_{n-2}^{2}(x) d x \geqslant \int_{-1}^{1}\left(1-x^{2}\right)^{2} q_{n-2}^{2}(x) d x \\
-\frac{1}{n} \int_{-1}^{1}\left(1-x^{2}\right)^{2} \sum_{k=1}^{n-2} \frac{\left(1-x_{k}^{2}\right)}{\left(x-x_{k}\right)^{2}} q_{n-2}^{2}(x) d x .
\end{array}
$$

IDENTITY 2.5. Let $P_{n}(x)$ be any polynomial of degree $n$; then we have 


$$
\begin{aligned}
(2 n+5)(2 n+1) \int_{-1}^{1} x^{4} P_{n}^{2}(x) d x+6 \int_{-1}^{1} x^{2} P_{n}^{2}(x) d x-3 \int_{-1}^{1} P_{n}^{2}(x) d x \\
=\frac{2(2 n+1)}{(n+2)} I_{2, n}+\frac{2(5 n+7)}{(n+1)(n+2)} I_{0, n} \\
-\frac{(5 n+7)}{(n+1)(n+2)} \int_{-1}^{1}\left(1-x^{2}\right)^{2} P_{n}^{2}(x) \sum_{k=1}^{n} \frac{\left(1-x_{k}^{2}\right)}{\left(x-x_{k}\right)^{2}} d x \\
-\frac{2(n+2)}{(n+1)} \int_{-1}^{1} x^{2}\left(1-x^{2}\right) \sum_{k=1}^{n} \frac{1-x_{k}^{2}}{\left(x-x_{k}\right)^{2}} P_{n}^{2}(x) d x,
\end{aligned}
$$

where

$$
I_{2 r, n}=\int_{-1}^{1} x^{2 r}\left(n x P_{n}(x)+\left(1-x^{2}\right) P_{n}^{\prime}(x)\right)^{2} d x .
$$

For the proof of this identity one uses (2.3) for $r=1$ and $r=2$.

Replacing $n$ by $n-2$ and $P_{n}(x)$ by $q_{n-2}(x)$, we obtain $(n>2)$

$$
\begin{array}{r}
4 n(n-1) \int_{-1}^{1} x^{4} q_{n-2}^{2}(x) d x \geqslant 3 \int_{-1}^{1}\left(1-x^{2}\right)^{2} q_{n-2}^{2}(x) d x \\
-\frac{(5 n-3)}{n(n-1)} \int_{-1}^{1}\left(1-x^{2}\right)^{2} \sum_{k=1}^{n-2} \frac{\left(1-x_{k}^{2}\right)}{\left(x-x_{k}\right)^{2}} q_{n-2}^{2}(x) d x \\
-\frac{2 n}{(n-1)} \int_{-1}^{1} x^{2}\left(1-x^{2}\right) \sum_{k=1}^{n-2} \frac{\left(1-x_{k}^{2}\right)}{\left(x-x_{k}\right)^{2}} q_{n-2}^{2}(x) d x .
\end{array}
$$

IDENTITY 2.6. Let $P_{n}(x)$ be any polynomial of degree $n$; then we have

$$
2 \int_{-1}^{1} x^{2} P_{n}^{\prime 2}(x) d x=\int_{-1}^{1} P_{n}^{2}(x) d x
$$

$$
\begin{aligned}
& +\int_{-1}^{1} x^{2}\left(P_{n}^{\prime 2}(x)-P_{n}(x) P_{n}^{\prime \prime}(x)\right) d x \\
& +\left(P_{n}(1) P_{n}^{\prime}(1)-P_{n}^{2}(1)\right)-\left(P_{n}(-1) P_{n}^{\prime}(-1)+P_{n}^{2}(-1)\right) .
\end{aligned}
$$

Proof. On integrating by parts, we obtain

$$
\begin{aligned}
\int_{-1}^{1} P_{n}^{2}(x) d x= & P_{n}^{2}(1)+P_{n}^{2}(-1)-\int_{-1}^{1} x \frac{d}{d x} P_{n}^{2}(x) d x \\
= & P_{n}^{2}(1)+P_{n}^{2}(-1)-\left(P_{n}(1) P_{n}^{\prime}(1)-P_{n}(-1) P_{n}^{\prime}(-1)\right) \\
& +\int_{-1}^{1} x^{2}\left(P_{n}^{\prime 2}(x)+P_{n}(x) P_{n}^{\prime \prime}(x)\right) d x .
\end{aligned}
$$

But this is equivalent to (2.22).

Lemma 2.1. Let $P_{n} \in H_{n}, P_{n}(1)=P_{n}(-1)=0$, and

$$
P_{n}(x)=\left(1-x^{2}\right) q_{n-2}(x),
$$

where $q_{n-2}(x)$ is a polynomial of degree $n-2$; then 


$$
\begin{aligned}
& \int_{-1}^{1} x^{2}\left(P_{n}^{\prime 2}(x)-P_{n}(x) P_{n}^{\prime \prime}(x)\right) d x \\
&>\left(\frac{1}{2}+\frac{3}{2(n-1)}\right) \int_{-1}^{1} P_{n}^{2}(x) d x \\
&-\frac{(n+1)}{2(n-1)^{2}} \int_{-1}^{1} P_{n}^{2}(x) \sum_{k=1}^{n-2} \frac{\left(1-x_{k}^{2}\right)}{\left(x-x_{k}\right)^{2}} d x,
\end{aligned}
$$

equality for $P_{n}(x)=\left(1-x^{2}\right)^{m}, n=2 m$.

Proof. On using (2.4)-(2.6) we obtain

$$
\begin{aligned}
\int_{-1}^{1} x^{2}\left(P_{n}^{\prime 2}(x)-\right. & \left.P_{n}(x) P_{n}^{\prime \prime}(x)\right) d x=\int_{-1}^{1} x^{2} P_{n}^{2}(x)\left(\sum_{k=1}^{n} \frac{1}{\left(x-x_{k}\right)^{2}}\right) d x \\
= & n \int_{-1}^{1} \frac{x^{2} P_{n}^{2}(x) d x}{\left(1-x^{2}\right)}-2 \int_{-1}^{1} \frac{P_{n}(x) P_{n}^{\prime}(x) x^{3}}{\left(1-x^{2}\right)} d x \\
& +\int_{-1}^{1} x^{2}\left(1-x^{2}\right) q_{n-2}^{2}(x) \sum_{k=1}^{n-2} \frac{\left(1-x_{k}^{2}\right)}{\left(x-x_{k}\right)^{2}} d x
\end{aligned}
$$

But

$$
\begin{aligned}
-\int_{-1}^{1} \frac{x^{3}}{\left(1-x^{2}\right)} \frac{d}{d x} P_{n}^{2}(x) d x= & 3 \int_{-1}^{1} \frac{x^{2}}{\left(1-x^{2}\right)} P_{n}^{2}(x) d x \\
& +2 \int_{-1}^{1} \frac{x^{4}}{\left(1-x^{2}\right)^{2}} P_{n}^{2}(x) d x
\end{aligned}
$$

Hence on using (2.20) and (2.21) we obtain

$$
\begin{aligned}
\int_{-1}^{1} x^{2}( & \left.P_{n}^{\prime 2}(x)-P_{n}(x) P_{n}^{\prime \prime}(x)\right) d x \\
= & (n+3) \int_{-1}^{1} x^{2}\left(1-x^{2}\right) q_{n-2}^{2}(x) d x \\
& +2 \int_{-1}^{1} x^{4} q_{n-2}^{2}(x) d x \\
& +\int_{-1}^{1} x^{2}\left(1-x^{2}\right) q_{n-2}^{2}(x) \sum_{k=1}^{n-2} \frac{1-x_{k}^{2}}{\left(x-x_{k}\right)^{2}} d x \\
\geqslant & \left(\frac{n+3}{2 n}+\frac{3}{2 n(n-1)}\right) \int_{-1}^{1} P_{n}^{2}(x) d x \\
& -\frac{(n+1)}{2(n-1)^{2}} \int_{-1}^{1} P_{n}^{2}(x) \sum_{k=1}^{n-2} \frac{\left(1-x_{k}^{2}\right)}{\left(x-x_{k}\right)^{2}} d x .
\end{aligned}
$$


3. Proof of Theorem 1. From (1.1) we have

$$
\begin{aligned}
2 \int_{-1}^{1}\left(1-x^{2}\right) P_{n}^{\prime 2}(x) d x= & n \int_{-1}^{1} P_{n}^{2}(x) d x \\
& +\int_{-1}^{1} P_{n}^{2}(x) \sum_{k=1}^{n-2} \frac{\left(1-x_{k}^{2}\right)}{\left(x-x_{k}\right)^{2}} d x
\end{aligned}
$$

(we used here $\left.P_{n}(1)=P_{n}(-1)=0\right)$. On using (2.22) and (2.23) we obtain

$$
\begin{aligned}
2 \int_{-1}^{1} x^{2} P_{n}^{\prime 2}(x) d x \geqslant & \int_{-1}^{1} P_{n}^{2}(x) d x+\left(\frac{1}{2}+\frac{3}{2(n-1)}\right) \int_{-1}^{1} P_{n}^{2}(x) d x \\
& -\frac{(n+1)}{2(n-1)^{2}} \int_{-1}^{1} P_{n}^{2}(x) \sum_{k=1}^{n-2} \frac{\left(1-x_{k}^{2}\right)}{\left(x-x_{k}\right)^{2}} d x
\end{aligned}
$$

On adding (3.1) and (3.2) we obtain $(n \geqslant 2)$

$$
\begin{aligned}
2 \int_{-1}^{1} P_{n}^{\prime 2}(x) d x \geqslant & \left(n+\frac{3}{2}+\frac{3}{2(n-1)}\right) \int_{-1}^{1} P_{n}^{2}(x) d x \\
& +\left(1-\frac{n+1}{2(n-1)^{2}}\right) \int_{-1}^{1} P_{n}^{2}(x) \sum_{k=1}^{n} \frac{\left(1-x_{k}^{2}\right)}{\left(x-x_{k}\right)^{2}} d x \\
\geqslant & \left(n+\frac{3}{2}-\frac{3}{2(n-1)}\right) \int_{-1}^{1} P_{n}^{2}(x) d x .
\end{aligned}
$$

This proves Theorem 1.

Proof of Theorem 2. Proof of (1.5)-(1.7) is similar. So we give the details for (1.5) only. From (2.1) and (2.13) we obtain

$$
\begin{aligned}
& 2 \int_{-1}^{1}\left(1-x^{4}\right) P_{n}^{\prime 2}(x) d x=(n+1) \int_{-1}^{1} P_{n}^{2}(x) d x \\
& \quad+(n-3) \int_{-1}^{1} x^{2} P_{n}^{2}(x) d x+S_{0}+S_{2} \\
& \geqslant\left(n+1+\frac{n-3}{2 n+3}\right) \int_{-1}^{1} P_{n}^{2}(x) d x \\
&+\frac{(n-3)\left(S_{2}-S_{0}\right)}{(n+1)(2 n+3)}+S_{0}+S_{2} \\
& \geqslant\left(n+\frac{3 n}{2 n+3}\right) \int_{-1}^{1} P_{n}^{2}(x) d x+\frac{2\left(n^{2}+2 n+3\right)}{(n+1)(2 n+3)} S_{0} \\
&+\frac{n(n+6)}{(n+1)(2 n+3)} S_{2} \\
& \geqslant\left(n+\frac{3 n}{2 n+3}\right) \int_{-1}^{1} P_{n}^{2}(x) d x .
\end{aligned}
$$

This proves Theorem 2 . 


\section{REFERENCES}

1. P. Turán, Über die Ablhingy wen Pabupmen, Compacitio Mhth 7 (1999) 09-95. MR 1, 37.

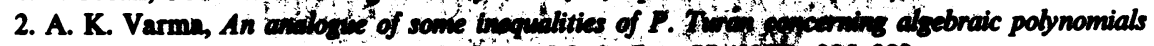
having all zeros inside $[-1,+1]$. Proe. Nitier. Math. Soc. 55 (19\%), 305-309.

Department of Mathematics, Universtty of Florida, GannesVILle, Florida 32611 